\title{
Risk-prediction tools for cardiovascular disease based on Japanese cohort studies
}

\author{
Tomonori Okamura and Aya Higashiyama \\ Hypertension Research (2009) 32, 1053-1054; doi:10.1038/hr.2009.166; published online 9 October 2009
}

$I^{\text {nom }}$ ndividuals in the general population would benefit from knowing their future risk of cardiovascular disease (CVD), such as coronary heart disease (CHD) or stroke, because this would motivate them to improve their lifestyle and/or take medication adequately. Many risk-prediction tools have been developed for this purpose, mainly in Western populations; for example, the Framingham CHD risk score and New Zealand and European risk-assessment charts have been used for patient education. ${ }^{1-4}$ High-risk strategy is an effective approach to preventing cardiovascular disease; high-risk status can be readily understood and strongly motivates individuals to change their lifestyle and/or maintain adherence to a medication regimen. Risk charts and scores are effective tools in such a strategy. ${ }^{5}$ However, we cannot adopt the risk charts developed among the foreign populations because of the difference in CVD mortality and its subtypes among different countries. ${ }^{6}$ Accordingly, there is a great need for risk-assessment charts or scores specific to the Japanese population.

A few risk charts and scores have been developed for Japanese populations. For example, in the National Integrated Project for Prospective Observation of Non-communicable Disease and Its Trends in the Aged, 1980 (NIPPON DATA80), Ueshima et al. ${ }^{6}$ presented their original risk charts, which were based on random sampling of the general population throughout Japan. ${ }^{7}$ In these charts, the probabilities of death within 10 years from CHD, stroke and all CVDs were compiled in a color-coded display combining 10-year age group, systolic blood pressure,

\footnotetext{
T Okamura and A Higashiyama are at the Department of Preventive Cardiology, National Cardiovascular Center, 5-7-1, Fujishirodai, Suita 565-8565, Japan.

E-mail: okamurat@hsp.ncvc.go.jp
}

smoking and serum total cholesterol and glucose levels. The six colors on each chart corresponded to levels of probability of CVD-related death. Unfortunately, these charts predicted only fatal CVD and were based on a limited number of predictive markers measured 30 years ago. The Japan Lipid Intervention Trial (J-LIT) charts also show absolute and relative risk for primary and secondary CHD, derived from a 6-year prospective study of about 50000 participants; however, the data are from a cohort of patients with mild hypercholesterolemia and under simvastatin treatment. ${ }^{8}$ Risk charts for CHD and stroke from the Jichi Medical School (JMS) cohort study are also available; these were based on a 10-year prospective study of about 12000 participants living in 12 communities, but the surveyed communities were limited to rural areas. ${ }^{9,10}$

Arima et al. ${ }^{11}$ have now developed a riskprediction tool based on one of the most famous Japanese cohorts. A total of 2634 participants in the Hisayama study were followed up for 14 years. Arima et al. ${ }^{11}$ used data from randomly selected two-thirds of the study population to develop a new risk-prediction model that was then tested to compare observed and predicted outcomes in the remaining one-third. The model showed good performance in detecting high-risk individuals. Data are collected using a simple risk score sheet that records sex, age, systolic blood pressure, low-density lipoprotein (LDL) cholesterol, high-density lipoprotein (HDL) cholesterol and smoking status. The model was the first to entail a score sheet rather than risk charts and to use LDL and HDL cholesterol levels as prediction markers in a community setting. This score sheet would be a useful guide in identifying individuals at high risk of CVD during annual health check-ups in rural communities.
Still, there is a need for additional prediction tools tailored to the Japanese population. Although the study by Arima et al. ${ }^{11}$ was carefully designed, it has some limitations, due mainly to its small sample size. First, sexspecific risk scores should be presented as in other previously published risk charts for the Japanese population; ${ }^{7-10}$ however, Arima et al. ${ }^{11}$ assessed men as having a higher risk for CVD than women by two pointsequivalent to the risk increase with 10 additional years of age. Second, two types of risk score sheets are needed: one for CHD and another for stroke. The risk score sheets of the Hisayama study combined these end points into one category. Many Japanese cohort studies, including the Hisayama study, have clearly shown, a high level of serum LDL cholesterol is a risk factor for CHD, but not for stroke. ${ }^{12,13}$ Third, their risk score sheet is based on a cohort study in one rural town. Accordingly, this score sheet may be limited in its generalizability to urban populations, in which the incidence of CHD is much higher than in rural populations. ${ }^{12,13}$

In any case, the risk-prediction tools available for use in the Japanese population will improve as ongoing epidemiological studies yield new outcomes. It is difficult to address this issue in a single cohort study. Metaanalysis using data on individual participants from many cohort studies may be an efficient way to develop a risk-prediction tool for the Japanese population in general. ${ }^{14}$

1 Wilson PWF, D'Agostino RB, Levy D, Belanger AM, Silbershatz H, Kannel WB. Prediction of coronary heart disease using risk factor categories. Circulation 1998; 97: 1837-1847.

2 Wood D, DeBacker G, Faergeman O, Graham I, Mancia G, Pyörälä K. Prevention of coronary heart disease in clinical practice. Recommendations of the Second Joint 
Task Force of European and other societies on coronary prevention. Eur Heart J 1998; 19: 1434-1503.

31996 National Heart Foundation clinical guidelines for the assessment and management of dyslipidaemia. Dyslipidaemia Advisory Group on behalf of the scientific committee of the National Heart Foundation of New Zealand. N Z Med J 1996; 109: 224-231.

4 Wallis EJ, Ramsay LE, Haq IU, Ghahramani P, Jackson PR, Rowlan-Yeo K, Rowland-Yeo K, Yeo WW. Coronary and cardiovascular risk estimation for primary prevention: validation of a new Sheffield table in the 1995 Scottish health survey population. BMJ 2000; 320: 671-676.

5 Rose G. Sick individuals and sick populations. Int J Epidemiol 1985; 14: 32-38.

6 Ueshima H, Sekikawa A, Miura K, Turin TC, Takashima N, Kita Y, Watanabe M, Kadota A, Okuda N, Kadowaki T, Nakamura Y, Okamura T. Cardiovascular disease and risk factors in Asia: a selected review. Circulation 2008; 118: 2702-2709.

7 NIPPON DATA80 Research Group. Risk assessment chart for death from cardiovascular disease based on a 19-year follow-up study of a Japanese representative population. Circ J 2006; 70: 1249-1255.

8 Mabuchi $H$. New guideline for the management of hyperlipidemia according to patient category and global risk chart. Nippon Rinsho 2002; 60: 908-915.

9 Matsumoto M, Ishikawa S, Kayaba K, Gotoh T, Nago N, Tsutsumi A, Kajii E, Jichi Medical School (JMS) Cohort Study Group. Risk charts illustrating the 10-year risk of myocardial infarction among residents of Japanese rural communities: the JMS Cohort Study. J Epidemiol 2009; 19: 94-100.

10 Ishikawa S, Matsumoto M, Kayaba K, Gotoh T, Nago N, Tsutsumi A, Kajii E, Jichi Medical School (JMS) Cohort Study Group. Risk charts illustrating the 10year risk of stroke among residents of Japanese rural communities: the JMS Cohort Study. J Epidemiol 2009; 19: 101-106.

11 Arima H, Yonemoto K, Doi Y, Ninomiya T, Hata J, Tanizaki Y, Fukuhara M, Matsumura K, lida M, Kiyohara Y. Development and validation of a cardiovascular risk prediction model for Japanese: The Hisayama Study. Hypertens Res 2009; 32: 1119-1122.

12 Okamura T, Kokubo Y, Watanabe M, Higashiyama A, Miyamoto Y, Yoshimasa Y, Okayama A. Low-densitylipoprotein cholesterol and non-high density lipoprotein cholesterol and the incidence of cardiovascular disease in an urban Japanese cohort study: the Suita study. Atherosclerosis 2009; 203: 587-592.

13 Imamura T, Doi Y, Arima H, Yonemoto K, Hata J, Kubo M, Tanizaki Y, Ibayashi S, Iida M, Kiyohara Y. LDL cholesterol and the development of stroke subtypes and coronary heart disease in a general Japanese population: the Hisayama study. Stroke 2009; 40: 382-388.

14 Murakami Y, Hozawa A, Okamura T, Ueshima H, Evidence for Cardiovascular Prevention From Observational Cohorts in Japan Research Group (EPOCH-JAPAN). Relation of blood pressure and all-cause mortality in 180,000 Japanese participants: pooled analysis of 13 cohort studies. Hypertension 2008; 51: 1483-1491. 